\title{
How can Canada safeguard those marginalized by society as MAiD expands?
}

\author{
Cite as: CMAJ 2021 April 6;193:E493-4. doi: 10.1503/cmaj.1095930
}

Posted on cmajnews.com on March 19, 2021

W hen Jeff Preston was hospitalized for pneumonia a few years ago, and a nurse asked if he wanted to be resuscitated if his heart or breathing stopped, he quipped that he wanted the care team to "do whatever is legally possible to keep me alive and then a few things after that."

Preston, an assistant professor of disability studies at Western University, has muscular dystrophy, and the nurse went on to describe in graphic detail the "undue suffering" he would experience during resuscitation and if he survived.

According to Preston, the nurse didn't coerce him or tell him what to do, but also didn't provide balanced information about how the care team would address his suffering if he wanted to be resuscitated. The message was clear: "Maybe, sometimes, it is best to just let go."

Devaluing encounters like this are the reason why Preston and others with disabilities are anxious about the recent expansion of medical assistance in dying (MAiD) in Canada. They fear that pervasive ableism and inadequate supports, especially in health care settings, will lead some people to seek death prematurely.

So far, Canada has restricted access to MAiD to people whose deaths were "reasonably foreseeable." But as the passage of Bill $\mathrm{C} 7$ has opened access to all adults who are experiencing intolerable suffering from a "grievous and irremediable" condition, Preston says tackling ableism will be an important next step to safeguard those most marginalized by society.

Some proponents of MAiD take issue with the notion that people with disabilities require safeguarding. According to Jocelyn Downie, a professor in the faculties of law

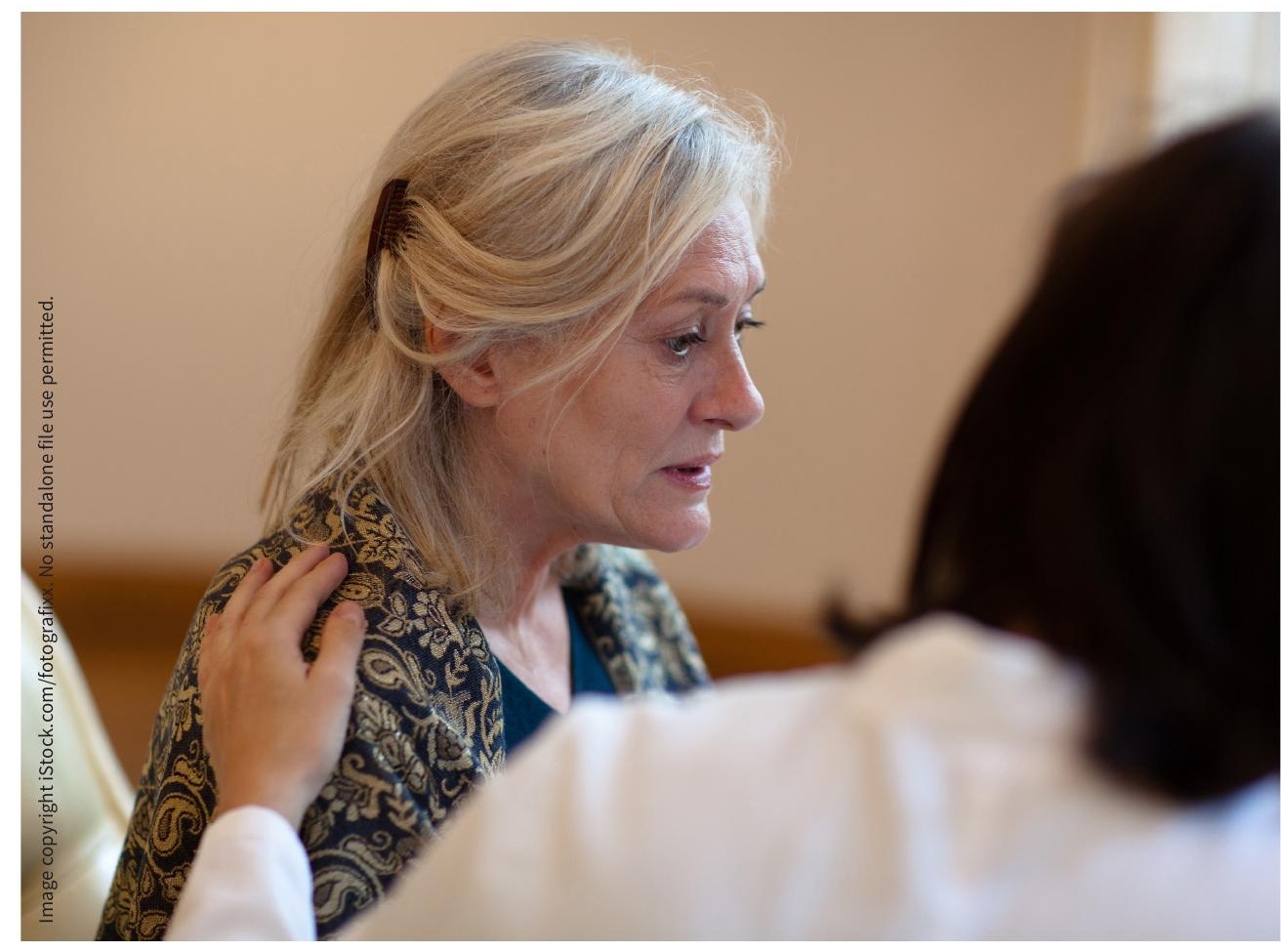

The passage of Bill $\mathrm{C} 7$ opens up access to medical assistance in dying, along with new challenges for health professionals.

and medicine at Dalhousie University, "the old legislation prevented them from accessing [MAiD] and was grounded in quite paternalistic concerns, basically protecting people from themselves."

Downie agrees that the expansion of MAiD should be paired with "improving access to mental health services and support for people with disabilities." Yet, she and others note that Canada's assessment process appears to be working well to screen people for the service.

"The process already protects people," says Helen Long, CEO of Dying with Dignity Canada. "To get MAiD, you have to make the request. You have to fill out the form [and] have it witnessed. You have to have two independent assessors either a doctor or a nurse practitioner, depending on the province. They assess your condition. They assess your pain and suffering. They talk to you about your values and beliefs."

According to the first annual report on MAiD in Canada, $99 \%$ of doctors who provided the service in 2019 had conversations with their patients to determine if their requests were voluntary. Three in four patients who requested MAiD received it. The most common reason for rejection related to patients' capacity to make health care decisions - concerns 
about coercion factored in less than $2 \%$ of rejections.

However, the reasons people sought MAiD were complicated. While most cited a loss of ability to engage in meaningful activities, and about half cited inadequate control of pain and other symptoms, more than a third reported feeling like a burden to loved ones. One in 10 reported isolation and loneliness as a factor in their decision.

\section{Tackling ableism}

While overt coercion may be rare, Preston and others say it's vital that health professionals understand and address how ableism contributes to patients' suffering. He says that includes ensuring that health workers embrace patients as equal members of the care team and provide unbiased information about all the options available to address suffering. "Lay out what else is out there - what the future might look like if you choose some of these options - and give people quality information in a way that they're able to understand."

Health workers tend to hold overly negative views about life with a disability, which can lead to discrimination in the treatment and referrals they provide. According to Erin Clark, a wheelchair user with congenital paralysis, patients often internalize these negative messages, too. "How do you hold your own life in esteem when you are explicitly told it is not valued by those you depend on to preserve it?"

Part of the problem is that many health professionals do not understand what life with a disability is like outside the medical context. Clark and others argue that doctors could benefit from learning from people with disabilities who are not their patients.

According to a CMAJ article by Heidi Janz, a specialist in disability ethics, "only if and when health care providers, educators and students are routinely and meaningfully exposed to the perspectives of people with disabilities can they develop a consistent proficiency in diagnosing and treating ableism."

\section{Defining irremediable}

Major questions also remain about assessing patients for MAiD when their sole underlying condition is mental illness.

The federal government originally intended to ban MAiD in such cases but agreed to lift that ban in two years after senators argued the exclusion was unconstitutional. In the meantime, the government will set up an expert panel to advise on safeguards and protocols that should apply to people with mental illnesses.

One key challenge will be defining "irremediable" in the context of mental disorders. "Ethically, as a provider, I hugely struggle with that," says Dr. Sonu Gaind, chief of psychiatry and medical director of mental health at Humber River Hospital in Toronto. "I could never say, based on any evidence, that I think your mental illness is irremediable. We simply can't know that."

Although studies demonstrate that at least some people do not respond to psychiatric treatment, there is no established standard of care for defining when a case should be considered irremediable, nor is there enough evidence yet to develop a standard.
According to a previous expert review commissioned by the federal government, "the issue is not whether there are people who have mental disorders that are irremediable, but rather whether clinicians can confidently determine whether a particular case is irremediable."

Because the course of many mental disorders fluctuates, there's no telling if someone who received MAiD might have improved, Gaind says. As such, it will be difficult to measure if safeguards are working, and approval decisions may vary by providers.

In one study of 66 cases of psychiatric euthanasia and assisted suicide in the Netherlands, assessors disagreed about patients' likelihood of improvement in one in five cases and delivered MAiD without resolving those disagreements.

Further complicating the picture, under Canadian law, patients are not required to exhaust treatment options to access MAiD, so clinicians will need a definition of "irremediable" that goes beyond a process of elimination. "If that safeguard can't be met, any of the [safeguards] after it make no difference," says Gaind.

Diana Duong, CMAJ, with files from Lauren Vogel

Content licence: This is an Open Access article distributed in accordance with the terms of the Creative Commons Attribution (CC BY-NC-ND 4.0) licence, which permits use, distribution and reproduction in any medium, provided that the original publication is properly cited, the use is noncommercial (i.e., research or educational use), and no modifications or adaptations are made. See: https://creativecommons.org/ licenses/by-nc-nd/4.0/ 